\title{
The RIT binary black hole simulations catalog
}

\author{
James Healy, Carlos O. Lousto, Yosef Zlochower, and Manuela Campanelli \\ Center for Computational Relativity and Gravitation, \\ School of Mathematical Sciences, Rochester Institute of Technology, \\ 85 Lomb Memorial Drive, Rochester, New York 14623
}

(Dated: March 13, 2017)

\begin{abstract}
The RIT numerical relativity group is releasing a public catalog of black-hole-binary waveforms. The initial release of the catalog consists of 126 recent simulations that include precessing and non precessing systems with mass ratios $q=m_{1} / m_{2}$ in the range $1 / 6 \leq q \leq 1$. The catalog contains information about the initial data of the simulation, the waveforms extrapolated to infinity, as well as information about the peak luminosity and final remnant black hole properties. These waveforms can be used to independently interpret gravitational wave signals from laser interferometric detectors and the remnant properties to model the merger of binary black holes from initial configurations.
\end{abstract}

PACS numbers: 04.25.dg, 04.25.Nx, 04.30.Db, 04.70.Bw

\section{INTRODUCTION}

The breakthroughs [1-3 in numerical relativity allowed numerical relativists to make detailed predictions for the gravitational waves from the latest inspiral, plunge, merger and ringdown of black hole binary systems (BHB). Numerical relativity predictions were confirmed by the first direct detection [4] of gravitational waves from such binary systems [5-7] and by its comparison to targeted runs [8, 9]. Those observations are consistent with general relativity as the correct theory for gravity as discussed in [7, 10].

The RIT group has been using numerical relativity techniques to explore the late dynamics of spinning blackhole binaries, beyond the post-Newtonian regime for many years. This includes simulations of the first generic, long-term precessing binary black hole evolution without any symmetry were performed in Ref. [11, where a detailed comparison with post-Newtonian $\ell=2,3$ waveforms was made, as well as studies of the hangup, i.e. the role individual black hole spins play to delay or accelerate their merger [12, the determination of the magnitude and direction of the recoil velocity of the final merged black hole [13 15, and the flip-flop of individual spins during the orbital phase [16 18]. Other numerical simulations have also explored the corners of parameter space, such as mass ratios $q=1 / 100$ in Ref. [19, and larger initial separations $R=100 M$ in [20]. And also near extremal $\chi=0.994$ spinning black hole binaries in 21] by the SXS collaboration.

There have been several significant efforts to coordinate numerical relativity simulations to support gravitational wave observations. These include the numerical injection analysis (NINJA) project 22 25, the numerical relativity and analytical relativity (NRAR) collaboration [26], and the waveform catalogs released by the simulating extreme spacetimes (SXS) collaboration [27] and Georgia Tech. [28].

The paper is organized as follows. Section II describe the methods and criteria for producing the numerical simulations. We next describe in Sec. [II] the use and content of the data in the public catalog. We conclude with a discussion in Sec. IV] of the use and potential extensions to this work to precessing binaries.

\section{FULL NUMERICAL EVOLUTIONS}

The runs in the RIT Catalog were evolved using the LAzEv [29] implementation of the moving puncture approach [2] with the conformal function $W=\sqrt{\chi}=$ $\exp (-2 \phi)$ suggested by Ref. 30]. In all cases we use the BSSNOK (Baumgarte-Shapiro-Shibata-NakamuraOohara-Kojima) family of evolutions systems [31 33. For the runs in the catalog, we used a variety of finitedifference orders, Kreiss-Oliger dissipation orders, and Courant factors 34$] 36$. Note that we do not upwind the advection terms. All of these are given in the metadata included in the catalog and the references associated with each run.

The LAzEv code uses the EinsteinToolkit [37, 38] / Cactus 39] / CARPet 40 infrastructure. The CARPET mesh refinement driver provides a "moving boxes" style of mesh refinement. In this approach, refined grids of fixed size are arranged about the coordinate centers of both holes. The CARPET code then moves these fine grids about the computational domain by following the trajectories of the two BHs.

We use AHFINDERDIRECT 41] to locate apparent horizons. We measure the magnitude of the horizon spin using the isolated horizon (IH) algorithm detailed in Ref. 42. and as implemented in Ref. 43]. Note that once we have the horizon spin, we can calculate the horizon mass via the Christodoulou formula $m_{H}=$ $\sqrt{m_{\mathrm{irr}}^{2}+S_{H}^{2} /\left(4 m_{\mathrm{irr}}^{2}\right)}$, where $m_{\mathrm{irr}}=\sqrt{A /(16 \pi)}, A$ is the surface area of the horizon, and $S_{H}$ is the spin angular momentum of the $\mathrm{BH}$ (in units of $M^{2}$ ). In the tables below, we use the variation in the measured horizon irreducible mass and spin during the simulation as a measure of the error in computing these quantities, since the lev- 
els of gravitational wave energy and momentum absorbed by the holes is orders of magnitude smaller. We measure radiated energy, linear momentum, and angular momentum, in terms of the radiative Weyl Scalar $\psi_{4}$, using the formulas provided in Refs. 44, 45. However, rather than using the full $\psi_{4}$, we decompose it into $\ell$ and $m$ modes and solve for the radiated linear momentum, dropping terms with $\ell>6$. The formulas in Refs. 44, 45, are valid at $r=\infty$. We extract the radiated energy-momentum at finite radius and extrapolate to $r=\infty$. We find that the new perturbative extrapolation described in Ref. 46] provides the most accurate waveforms. While the difference of fitting both linear and quadratic extrapolations provides an independent measure of the error. Studies of the finite difference errors, and verification that the waveforms provided in this catalog are computed at a numerical resolution in the convergence regime can be found in the appendices of Refs. 47] and [36. Although higher multipoles modes [beyond $(\ell, m)=(2, \pm 2)$ ] are not provided in the catalog for the sake of simplicity, they are used (typically up to $\ell=4$ or $\ell=6$ ) in the computation of radiative quantities such as the energy and linear and angular momenta.

To compute the initial low eccentricity orbital parameters we use the post-Newtonian techniques described in [48. To compute the numerical initial data, we use the puncture approach [49] along with the TwoPunCTURES [50] thorn.

We measure the distance between the two BHs using the simple proper distance or SPD. The SPD is the proper distance, on a given spatial slice, between the two $\mathrm{BH}$ apparent horizons as measured along the coordinate line joining the two centers. As such, it is gauge dependent, but still gives reasonable results (see Ref. 20] for more details).

\section{CATALOG}

The RIT Catalog can be found at http://ccrg.rit. edu/ RITCatalog. Figure 1 shows the distribution of non-precessing runs in the catalog in terms of $\chi_{12}$ and $q$ ( $\chi$ here is the $z$-component of the dimensionless spin). The information currently in the catalog consists of the metadata describing the runs and $(\ell=2, m= \pm 2)$ modes of $r \psi_{4}$. The extrapolations of $r \psi_{4}$ to $r=\infty$ are performed using the perturbative approach of [46]. The associated metadata include the initial orbital frequencies, ADM masses, initial waveform frequencies, black hole masses, momenta, spins, separations, and eccentricities, as well the black-hole masses and spins once the initial burst of radiation has left the region around the binary. These relaxed quantities are more accurate for modeling purposes than the initial masses and spins. In addition, we also include the final remnant masses and spins.

The catalog is organized using an interactive table that includes an identification number, resolution, type of run

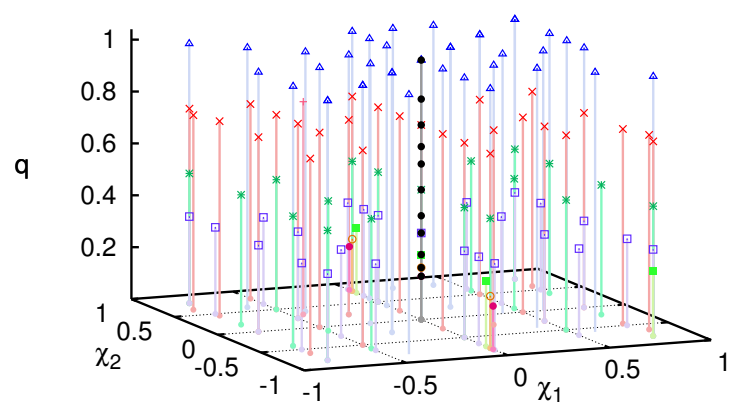

FIG. 1. Initial parameters in the $\left(q, \chi_{1}, \chi_{2}\right)$ space for the 120 nonprecessing binaries. Each mass ratio is given a different color: blue triangles $(q=1.00)$, pink plus $(q=0.82)$, red crosses $(q=3 / 4)$, green stars $(q=1 / 2)$, purple squares $(q=$ $1 / 3)$, light green full squares $(q=1 / 4)$, orange $\operatorname{circles}(q=$ $1 / 5)$, and brown full circles $(q=1 / 6)$. Nonspinning runs are black full circles.
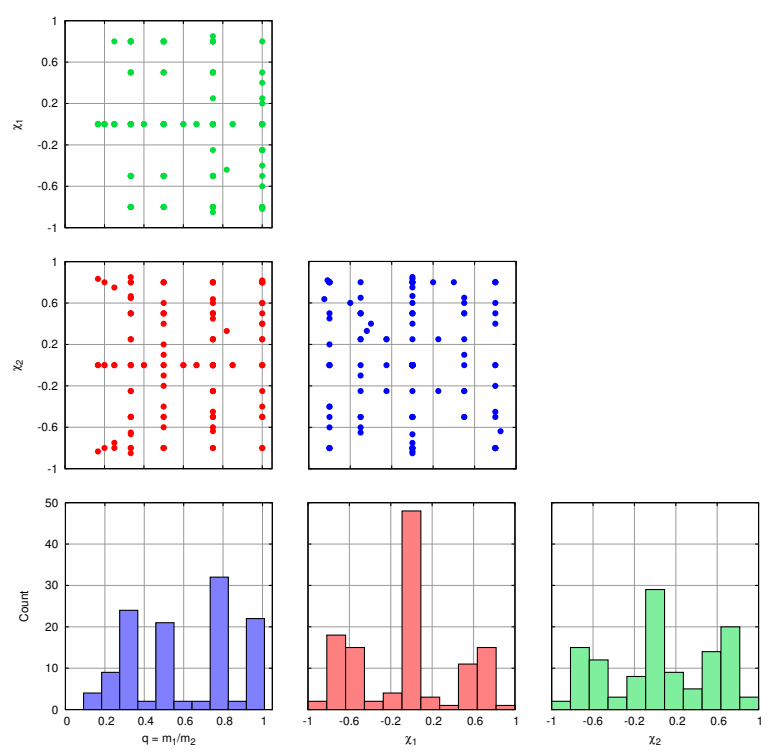

FIG. 2. Initial parameters in the $\left(q, \chi_{1}, \chi_{2}\right)$ plane for the 120 nonprecessing binaries. Note that here $q=m_{1} / m_{2}<1$.

(nonspinning, aligned spins, precessing), the initial simple proper distance between the two black holes, the coordinate separation, the mass ratio of the two black holes, the components of the dimensionless spins of the two black holes, the starting waveform frequency, time to merger, number of gravitational wave cycles, remnant mass, remnant spin, recoil velocity, and peak luminosity. The final column gives the appropriate bibtex keys for the relevant publications where the waveforms were first presented. The table can be sorted (ascending or descending) by any of these columns. 


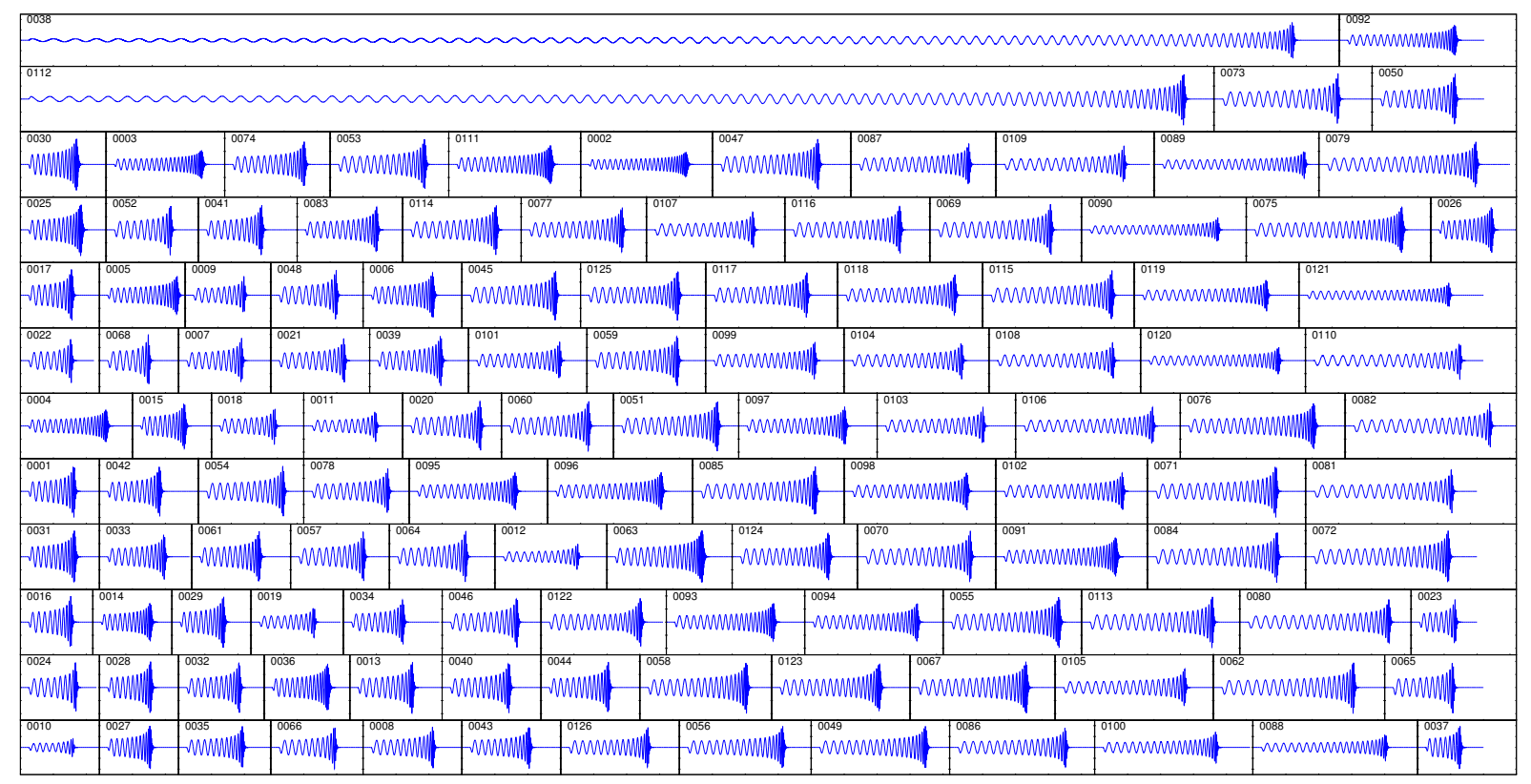

FIG. 3. Visual display of the different lengths of the $(2,2)$ waveforms in this first delivery of 126 simulations in the RIT Catalog. Each row of waveforms spans $\sim 22700 M$ of simulation time from edge to edge, with each tic mark denoting $500 M$.

The initial waveform frequency, denoted by $M f_{22 \text {,start }}$ in the table gives the starting frequency in units of $2.03 \times$ $10^{5}\left(\frac{M_{\odot}}{M}\right) \mathrm{Hz}$, where $M$ is the mass of the binary and $M_{\odot}$ is one solar mass (e.g., $M f_{22 \text {,start }}=0.01$ corresponds to $34 \mathrm{~Hz}$ for a $60 M_{\odot}$ binary). Note that $2 \pi f_{22}=\omega_{22}$. The runs in the catalog span initial frequencies from 0.003 to 0.012 , with a corresponding initial proper separations of $10.59 \mathrm{M}$ to $25.18 \mathrm{M}$. Times from the start of the simulation to merger range from $556 \mathrm{M}$ to $19219 \mathrm{M}$, and the number of inspiral cycles in the $(\ell=2, m=2)$ mode of $\psi_{4}$ range from 8.3 to 89.9 .

Resolutions are given in terms of the gridspacing of the refinement level where the waveform is extracted (which is typically two refinement levels below the coarsest grid) with $R_{o b s} \sim 100 M$. We use the notation nXYY, where the gridspacing in the wavezone is given by $\mathrm{M} / \mathrm{X}$.YY, e.g., n120 corresponds to $h=M / 1.2$.

For each simulation in the catalog there are two files: one contains the metadata information in ASCII format, the other is a tar.gz file containing ASCII files with the $(\ell=2, m= \pm 2)$ modes of $r \psi_{4}$ (extrapolated to $\left.r=\infty\right)$.

Note that our catalog provides the Weyl scalar $r \psi_{4}$ extrapolated to infinity rather than the strain $\hat{h}$. We leave to the user to convert $r \psi_{4}$ to strain (e.g., along the lines of the method delineated in the NRAR collaboration [26], for instance).

Figure 2 shows the distribution of the 120 nonprecessing runs in the catalog in terms of $\chi_{1,2}$ and $q(\chi$ here is the $L$ or $z$-component of the dimensionless spin).

\section{CONCLUSIONS AND DISCUSSION}

The breakthroughs [1] in numerical relativity were instrumental in identifying the first detection of gravitational waves [4] with the merger of two black holes. The direct comparison of numerical waveforms with observations also allows one to determine the parameters of such binary [8. The current catalog of waveforms as displayed in Fig. 3 can be used to perform independent analysis by the wider gravitational wave community and serves as a platform to deliver new sets of simulations as they become available.

Aside the interest in producing waveforms for direct comparison with observation, the simulations of orbiting black hole binaries produce information about the final remnant of the merger of the two holes. This was already the subject of early studies using the Lazarus approach [51, 52. With the advent of the breakthroughs that allowed for longer accurate computations, numerous empirical formulas relating the initial parameters $\left(q, \vec{\chi}_{1}, \vec{\chi}_{2}\right)$ (individual masses and spins) of the binary to those of the final remnant $\left(m_{f}, \vec{\chi}_{f}, \vec{V}_{f}\right)$ have been proposed. These include formulas for the final mass, spin, and recoil velocity [47, 53 59, as well as algebraic properties of the final metric 60, 61. Recently, the computation of the peak luminosity has also been the subject of interest in relation to the observation of gravitational waves [4, 7, 36, 62. The data in RIT catalog, along with the SXS 27] and Georgia Tech. 28, can be used by other groups to develop and improve new empirical formulas for the remnant properties and approxi- 
mate/phenomenological waveform models [63, 64].

\section{ACKNOWLEDGMENTS}

The authors thank N.K.J-McDaniel, H. Nakano, and R. O'Shaughnessy for discussions on this work. The authors gratefully acknowledge the NSF for financial support from NSF Grants No. PHY-1607520, No.
ACI-1550436, No. AST-1516150, No. ACI-1516125, No. PHY-1305730, No. PHY-1212426, No. PHY1229173, No. AST-1028087, No. PHY-0969855, No. OCI-0832606, and No. DRL-1136221. Computational resources were provided by XSEDE allocation TGPHY060027N, and by NewHorizons and BlueSky Clusters at Rochester Institute of Technology, which were supported by NSF grants No. PHY-0722703, No. DMS0820923, No. AST-1028087, and No. PHY-1229173.
[1] F. Pretorius, Phys. Rev. Lett. 95, 121101 (2005), grqc/0507014

[2] M. Campanelli, C. O. Lousto, P. Marronetti, and Y. Zlochower, Phys. Rev. Lett. 96, 111101 (2006), grqc/0511048

[3] J. G. Baker, J. Centrella, D.-I. Choi, M. Koppitz, and J. van Meter, Phys. Rev. Lett. 96, 111102 (2006), grqc/0511103

[4] B. P. Abbott et al. (Virgo, LIGO Scientific), Phys. Rev. Lett. 116, 241102 (2016), arXiv:1602.03840 [gr-qc]

[5] B. Abbott et al. (Virgo, LIGO Scientific), Phys. Rev. Lett. 116, 061102 (2016), arXiv:1602.03837 [gr-qc]

[6] B. P. Abbott et al. (Virgo, LIGO Scientific), Phys. Rev. Lett. 116, 241103 (2016), arXiv:1606.04855 [gr-qc]

[7] B. P. Abbott et al. (Virgo, LIGO Scientific), Phys. Rev. X6, 041015 (2016), arXiv:1606.04856 [gr-qc]

[8] B. P. Abbott et al. (Virgo, LIGO Scientific), Phys. Rev. D94, 064035 (2016), arXiv:1606.01262 [gr-qc]

[9] G. Lovelace et al., Class. Quant. Grav. 33, 244002 (2016). arXiv:1607.05377 [gr-qc].

[10] B. P. Abbott et al. (Virgo, LIGO Scientific), Phys. Rev. Lett. 116, 221101 (2016), arXiv:1602.03841 [gr-qc]

[11] M. Campanelli, C. O. Lousto, H. Nakano, and Y. Zlochower, Phys. Rev. D79, 084010 (2009), arXiv:0808.0713 [gr-qc]

[12] M. Campanelli, C. O. Lousto, and Y. Zlochower, Phys. Rev. D74, 041501(R) (2006), gr-qc/0604012.

[13] M. Campanelli, C. O. Lousto, Y. Zlochower, and D. Merritt, Astrophys. J. 659, L5 (2007), gr-qc/0701164

[14] M. Campanelli, C. O. Lousto, Y. Zlochower, and D. Merritt, Phys. Rev. Lett. 98, 231102 (2007), gr-qc/0702133

[15] C. O. Lousto and Y. Zlochower, Phys. Rev. Lett. 107, 231102 (2011), arXiv:1108.2009 [gr-qc].

[16] C. O. Lousto and J. Healy, Phys. Rev. Lett. 114, 141101 (2015), arXiv:1410.3830 [gr-qc]

[17] C. O. Lousto, J. Healy, and H. Nakano, Phys. Rev. D93, 044031 (2016), arXiv:1506.04768 [gr-qc]

[18] C. O. Lousto and J. Healy, Phys. Rev. D93, 124074 (2016), arXiv:1601.05086 [gr-qc]

[19] C. O. Lousto and Y. Zlochower, Phys. Rev. Lett. 106, 041101 (2011), arXiv:1009.0292 [gr-qc].

[20] C. O. Lousto and Y. Zlochower, Phys. Rev. D88, 024001 (2013), arXiv:1304.3937 [gr-qc]

[21] G. Lovelace, M. A. Scheel, R. Owen, M. Giesler, R. Katebi, B. Szilágyi, T. Chu, N. Demos, D. A. Hemberger, L. E. Kidder, H. P. Pfeiffer, and N. Afshari, Class. Quant. Grav. 32, 065007 (2015), arXiv:1411.7297 [gr-qc].

[22] B. Aylott et al., Class. Quant. Grav. 26, 165008 (2009) arXiv:0901.4399 [gr-qc].
[23] B. Aylott et al., Class. Quant. Grav. 26, 114008 (2009) arXiv:0905.4227 [gr-qc].

[24] P. Ajith et al., Class. Quant. Grav. 29, 124001 (2012) arXiv:1201.5319 [gr-qc].

[25] J. Aasi et al. (LIGO Scientific Collaboration, Virgo Collaboration, NINJA-2 Collaboration), Class. Quant. Grav. 31, 115004 (2014), arXiv:1401.0939 [gr-qc]

[26] I. Hinder, A. Buonanno, M. Boyle, Z. B. Etienne, J. Healy, N. K. Johnson-McDaniel, A. Nagar, H. Nakano, Y. Pan, H. P. Pfeiffer, M. Pürrer, C. Reisswig, M. A. Scheel, E. Schnetter, U. Sperhake, B. Szilágyi, W. Tichy, B. Wardell, A. Zenginoğlu, D. Alic, S. Bernuzzi, T. Bode, B. Brügmann, L. T. Buchman, M. Campanelli, T. Chu, T. Damour, J. D. Grigsby, M. Hannam, R. Haas, D. A. Hemberger, S. Husa, L. E. Kidder, P. Laguna, L. London, G. Lovelace, C. O. Lousto, P. Marronetti, R. A. Matzner, P. Mösta, A. Mroué, D. Müller, B. C. Mundim, A. Nerozzi, V. Paschalidis, D. Pollney, G. Reifenberger, L. Rezzolla, S. L. Shapiro, D. Shoemaker, A. Taracchini, N. W. Taylor, S. A. Teukolsky, M. Thierfelder, H. Witek, and Y. Zlochower, Class. Quant. Grav. 31, 025012 (2014), arXiv:1307.5307 [gr-qc],

[27] A. H. Mroue, M. A. Scheel, B. Szilagyi, H. P. Pfeiffer, M. Boyle, et al., Phys. Rev. Lett. 111, 241104 (2013) arXiv:1304.6077 [gr-qc]

[28] K. Jani, J. Healy, J. A. Clark, L. London, P. Laguna, and D. Shoemaker, Class. Quant. Grav. 33, 204001 (2016) arXiv:1605.03204 [gr-qc]

[29] Y. Zlochower, J. G. Baker, M. Campanelli, and C. O. Lousto, Phys. Rev. D72, 024021 (2005), arXiv:grqc/0505055

[30] P. Marronetti, W. Tichy, B. Brügmann, J. Gonzalez, and U. Sperhake, Phys. Rev. D77, 064010 (2008), arXiv:0709.2160 [gr-qc]

[31] T. Nakamura, K. Oohara, and Y. Kojima, Prog. Theor. Phys. Suppl. 90, 1 (1987).

[32] M. Shibata and T. Nakamura, Phys. Rev. D52, 5428 (1995).

[33] T. W. Baumgarte and S. L. Shapiro, Phys. Rev. D59, 024007 (1998), gr-qc/9810065

[34] C. O. Lousto and Y. Zlochower, Phys. Rev. D77, 024034 (2008), arXiv:0711.1165 [gr-qc]

[35] Y. Zlochower, M. Ponce, and C. O. Lousto, Phys. Rev. D86, 104056 (2012), arXiv:1208.5494 [gr-qc].

[36] J. Healy and C. O. Lousto, Phys. Rev. D95, 024037 (2017), arXiv:1610.09713 [gr-qc].

[37] F. Löffler, J. Faber, E. Bentivegna, T. Bode, P. Diener, R. Haas, I. Hinder, B. C. Mundim, C. D. Ott, E. Schnetter, G. Allen, M. Campanelli, and P. Laguna, Class. Quant. Grav. 29, 115001 (2012), arXiv:1111.3344 [gr-qc] 
[38] Einstein Toolkit home page: http://einsteintoolkit.org.

[39] Cactus Computational Toolkit home page: http://cactuscode.org.

[40] E. Schnetter, S. H. Hawley, and I. Hawke, Class. Quant. Grav. 21, 1465 (2004), gr-qc/0310042.

[41] J. Thornburg, Class. Quant. Grav. 21, 743 (2004), grqc/0306056

[42] O. Dreyer, B. Krishnan, D. Shoemaker, and E. Schnetter, Phys. Rev. D67, 024018 (2003), gr-qc/0206008

[43] M. Campanelli, C. O. Lousto, Y. Zlochower, B. Krishnan, and D. Merritt, Phys. Rev. D75, 064030 (2007), gr-qc/0612076

[44] M. Campanelli and C. O. Lousto, Phys. Rev. D59, 124022 (1999), arXiv:gr-qc/9811019 [gr-qc]

[45] C. O. Lousto and Y. Zlochower, Phys. Rev. D76, 041502(R) (2007), gr-qc/0703061.

[46] H. Nakano, J. Healy, C. O. Lousto, and Y. Zlochower, Phys. Rev. D91, 104022 (2015), arXiv:1503.00718 [grqc].

[47] J. Healy, C. O. Lousto, and Y. Zlochower, Phys. Rev. D90, 104004 (2014), arXiv:1406.7295 [gr-qc].

[48] J. Healy, C. O. Lousto, H. Nakano, and Y. Zlochower, (2017), arXiv:1702.00872 [gr-qc]

[49] S. Brandt and B. Brügmann, Phys. Rev. Lett. 78, 3606 (1997), gr-qc/9703066.

[50] M. Ansorg, B. Brügmann, and W. Tichy, Phys. Rev. D70, 064011 (2004), gr-qc/0404056.

[51] J. G. Baker, M. Campanelli, C. O. Lousto, and R. Takahashi, Phys. Rev. D69, 027505 (2004), arXiv:astro$\mathrm{ph} / 0305287$.
[52] M. Campanelli, Class. Quant. Grav. 22, S387 (2005), astro-ph/0411744.

[53] E. Barausse, V. Morozova, and L. Rezzolla, Astrophys. J. 758, 63 (2012), arXiv:1206.3803 [gr-qc],

[54] L. Rezzolla, E. Barausse, E. N. Dorband, D. Pollney, C. Reisswig, J. Seiler, and S. Husa, Phys. Rev. D78, 044002 (2008), arXiv:0712.3541 [gr-qc],

[55] F. Hofmann, E. Barausse, and L. Rezzolla, Astrophys. J. 825, L19 (2016), arXiv:1605.01938 [gr-qc],

[56] X. Jiménez-Forteza, D. Keitel, S. Husa, M. Hannam, S. Khan, and M. Pürrer, (2016), arXiv:1611.00332 [grqc]

[57] C. O. Lousto, M. Campanelli, Y. Zlochower, and H. Nakano, Class. Quant. Grav. 27, 114006 (2010), arXiv:0904.3541 [gr-qc],

[58] C. O. Lousto and Y. Zlochower, Phys. Rev. D89, 104052 (2014), arXiv:1312.5775 [gr-qc]

[59] Y. Zlochower and C. O. Lousto, Phys. Rev. D92, 024022 (2015), arXiv:1503.07536 [gr-qc]

[60] M. Campanelli, C. O. Lousto, and Y. Zlochower, Phys. Rev. D79, 084012 (2009), arXiv:0811.3006 [gr-qc].

[61] R. Owen, Phys. Rev. D81, 124042 (2010), arXiv:1004.3768 [gr-qc].

[62] D. Keitel et al., (2016), arXiv:1612.09566 [gr-qc],

[63] S. Babak, A. Taracchini, and A. Buonanno, Phys. Rev. D95, 024010 (2017), arXiv:1607.05661 [gr-qc],

[64] S. Khan, S. Husa, M. Hannam, F. Ohme, M. Prrer, X. Jimnez Forteza, and A. Boh, Phys. Rev. D93, 044007 (2016), arXiv:1508.07253 [gr-qc] 